\title{
A systematized approach to AVHRR image navigation
}

\author{
D. G. BALDWIN AND W.J. EMERY \\ Campus Box 429, Cooperative Institute for Research in Environmental Sciences, \\ University of Colorado at Boulder, CO 80309-0216, U.S.A.
}

\begin{abstract}
Accurate co-location and geo-registration of AVHRR (Advanced Very High Resolution Radiometer) is necessary for most applications involving these data. To obtain the necessary accuracy, most of the current systems available for AVHRR image rectification require corrections to errors arising from several different sources. This paper presents a software package which is able to correct registration errors from all sources using effective roll, pitch, and yaw values determined from ground control point offsets. These effective values are used to establish a baseline registration against which other models are compared. Spacecraft and sampling geometry are revisited in order to systemize and better define the procedure of AVHRR geo-registration. This systematic approach has resulted in a modular software package which has a registration accuracy of 1 satellite pixel, and is easily modified and improved.
\end{abstract}

\section{INTRODUCTION}

As recently reviewed by Emery and others (1989), the navigation of imagery from the Advanced Very High Resolution Radiometer(AVHRR) has developed significantly over the past decade. Simple "forward navigation" algorithms of the past have evolved to the more sophisticated "indirect navigation" systems of today. The former use orbital models to distort geographic overlays to fit images in "satellite perspective" while the latter use orbital models to co-locate satellite data to fit a specified geographic projection.

The problem of image navigation can be split into two basic components. The first consists of making the proper corrections for geometric distortions that occur due to the oblateness of the earth and the non-linear distribution of the measurements taken by the sensor. Brush (1987) gives a good description of these distortions.

The second component involves corrections for errors which arise from non-geometrical sources. These include satellite timing and positioning errors, errors in satellite altitude, and misalignment of the sensor's axes (roll, pitch and yaw). Generally some combination of ephemerisdriven orbital models, timing corrections and ground control point (GCP) offsets are used to compensate for these. Ho and Asem (1986), Brunel and Marsouin (1987), and Emery and Ikeda (1984) present different methods of solving the navigation problem. Several of these methods are summarized and compared in Emery and others (1989).

This paper presents a method which uses an accurate ephemeris-driven orbital model to properly position the satellite, and the offsets of two GCPs to compute an "effective" roll, pitch and yaw. This correction compensates for errors from all sources and results in registration accuracies of $1 \mathrm{~km}$. Furthermore, the effective corrections can be used to establish a baseline registration against which other models may be compared. This correction method has initiated a follow up study, currently in progress, which is investigating the potential of modelling the effective corrections, thus greatly reducing the need for adjusting to GCPs. This will provide an obvious advantage to researchers using ocean data which contains no landmarks for GCPs.

In order to systemize the navigation procedure, we have revisited the spacecraft and sampling geometry to better define and apply the orbital model. Here we present a system for the creation of geo-registered images from AVHRR data. The ephemeris-driven orbital model and the effective roll, pitch and yaw corrections are both outlined, as are procedures for co-locating the data using indirect navigation. Some results using the navigation system are discussed and a comparison between different orbital model configurations is presented.

A detailed mathematical presentation of the theory involved in this system is beyond the scope of this discussion; however qualitative outlines of the procedures and mechanics are illustrated whenever necessary. The mathematical models used for orbit and attitude determination and indirect navigation are presented in Rosborough and others (in preparation).

\section{TIROS AND AVHRR GEOMETRY}

The AVHRR sensor is carried on the TIROS-series spacecraft (TIROS-N, NOAA6, NOAA7 ... NOAA12) which is in a near-circular sun-synchronous orbit at a nominal altitude of $850 \mathrm{~km}$. The TIROS series of spacecraft are three-axis stabilized and attempt to 
maintain a constant reference attitude (roll,pitch and yaw) at all times. This reference attitude corresponds to one axis pointing to nadir and another axis being maintained perpendicular to the inertial velocity vector of the satellite. AVHRR scanning occurs on a plane defined by these two axes. Deviations of the axes from the reference attitude are defined as roll,pitch and yaw. Roll is the angular deviation occurring in the scanning plane, pitch is the deviation along the satellites track and yaw is the deviation in the plane which is perpendicular to the nadir pointing axis.

The AVHRR system consists of a five-channel radiometer. One of the channels measures light in the visible wavelength band, another in the near-infrared, and the remaining three measure emitted radiation in the infrared wavelengths. The bandwidths for the five channels of the instrument on board NOAA-11 are presented in Table 1 .

There are 2048 measurements made each scan and 6 scans are made every second. A scanning pass is referred to as a "scan line" and the individual measurements are called "samples". The scan line and sample numbers define the field of view (FOV) or individual measurement of a given channel. At the nadir point, the sensor measures a $1.1 \mathrm{~km}^{2}$ region: this increases to approximately $6.8 \mathrm{~km}^{2}$ at the limbs of the scan. Further details describing the satellite and AVHRR sensor are contained in Brush (1982).

\section{EPHEMERIS, ORBITAL MODEL AND SATELLITE POSITION}

The navigation algorithms require the position and inertial velocity of the spacecraft expressed in an Earthfixed coordinate system. These quantities are determined from the classical orbital elements predicted by an orbital model. Typically the orbital model predicts these elements from differential equations with initial conditions obtained from a set of ephemeris elements for the particular satellite.

Usually the orbital model is specific to the type of ephemeris data which are used so the design of our navigational package is such that any appropriate

Table 1. Bandwidths for NOAA-11 channels 1-5

\section{Channel Bandwidth}

$\mu \mathrm{m}$,

above $10 \%$ normalized response

$\begin{array}{cc}1 & 0.5-0.7 \\ 2 & 0.7-1.0 \\ 3 & 3.5-4.0 \\ 4 & 10.2-11.4 \\ 5 & 11.3-12.5\end{array}$

combination of ephemeris and orbital models can be implemented. In the current configuration, ephemeris and orbital models supplied by the U.S. Naval Space Surveillance Center (NAVSPASUR) are used. The orbital elements are supplied daily for each of the current NOAA satellites and are valid at a certain instant of time defined as the ephemeris epoch.

As mentioned above, the ephemeris elements are used to construct initial condition inputs to the orbital model. These inputs consist of the semi-major axis, eccentricity, inclination, argument of perigee, longitude of ascending node, and mean anomaly. It will further be assumed that the secular decay of the semi-major axis due to atmospheric drag is also known. Given this information at epoch, it is possible to predict these elements at some other time by assuming that the orbit is a secularlyprecessing decaying ellipse.

The secular motion is due to the earth oblateness and causes linear changes in time of the argument of perigee, longitude of ascending node and mean anomaly. The decay causes a linear change in the semi-major axis as well as an indirect effect on the secular motions. Short period perturbations, known as osculating perturbations, are corrected using the Brouwer method (Brouwer, 1959). In summary, the orbital model propagates the orbital elements from the epoch time to some time in the future. The elements can then be used to construct geocentric cartesian vectors which describe the spacecrafts position and velocity at the time of prediction.

\section{INDIRECT NAVIGATION}

Indirect navigation is the procedure by which satellite data are resampled to fit a predefined geographic grid, thus a point on the earth is mapped to a satellite FOV.

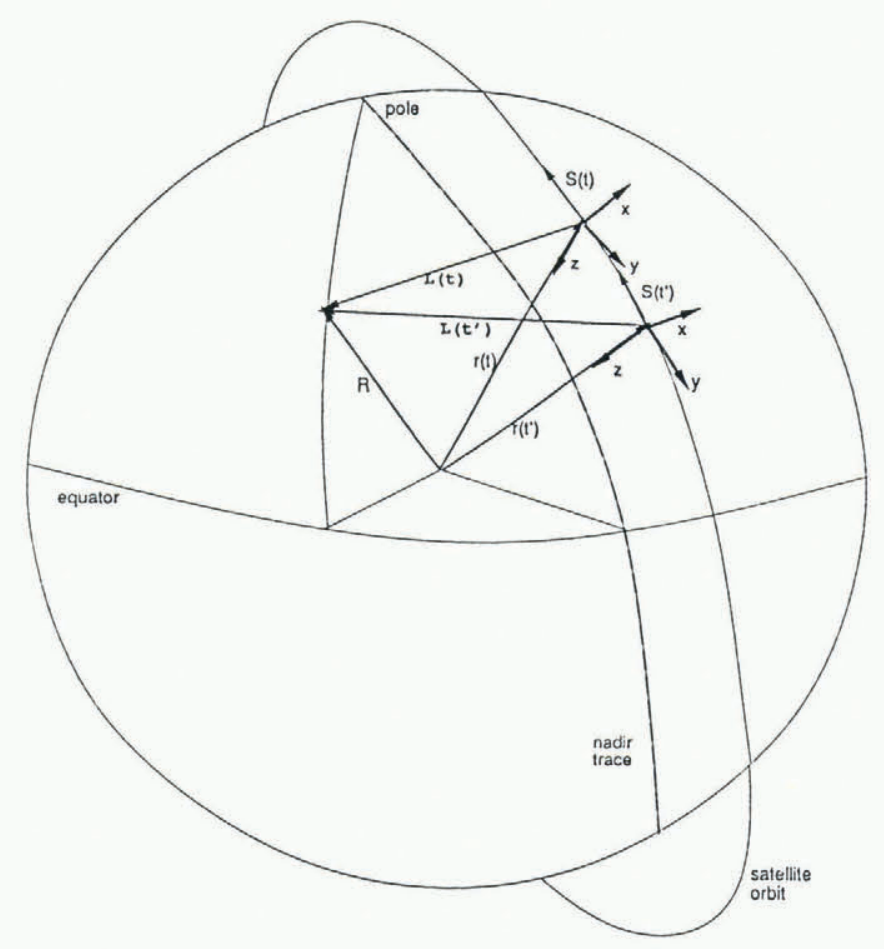

Fig. 1. Orbital and scanning geometry at solution time $t$ and intermediate time $t^{\prime}$. 
This is in contrast to the direct method which maps the satellite FOV to an earth location. The indirect method is more desirable since it results in the mapping of every image pixel and, in the full resolution case, every satellite measurement. The steps required to create a georegistered image from AVHRR data using indirect navigation techniques are described below, the corresponding geometry is illustrated in Figure 1.

(1) The geometry of the image is specified by choosing an output image size in pixels, a center latitude and longitude for the image, a latitude range, and a geographic projection. Once these parameters have been chosen, a mapping has been established which assigns a latitude and longitude to each pixel in the image.

(2) Each latitude and longitude is then mapped to the corresponding FOV and the appropriate satellite measurement is retrieved. As mentioned before, the FOV is defined by the scan line and sample number within the data.

Step 1 is a simple mathematical exercise in geographic projections, while step 2 requires more complicated procedures which are qualitatively outlined below.

The time $(t)$ at which the particular point (defined by the latitude, longitude) was sampled is unknown and must be determined through an iterative procedure. Briefly, a geocentric vector $\mathbf{R}$ is drawn to the point and a first guess estimate $\left(t^{\prime}\right)$ is made for the time of observation. The first guess can be either the time of first scan of the data, or the time of the previous solution. The orbital elements are predicted at $t^{\prime}$ and vectors describing the position, $\mathbf{r}\left(t^{\prime}\right)$, and velocity $\mathbf{S}\left(t^{\prime}\right)$, of the spacecraft are calculated. The "look" vector $\mathbf{L}\left(t^{\prime}\right)$, pointing from the spacecraft to the defined point is constructed and transformed to a satellite-centered coordinate system using a transformation matrix defined by the position and velocity of the spacecraft.

The satellite-based system is orthogonal, with the $y$ unit vector pointing opposite the satellite's velocity, the $z$ unit vector pointing towards nadir and the $\mathrm{x}$ unit vector oriented perpendicular to the $y-z$ plane. A vector with components representing the effective roll, pitch and yaw is applied during this transformation to properly orient the resulting look vector.

If the time estimate $t^{\prime}$ was correct, $\mathrm{L}\left(t^{\prime}\right)$ would lie in the $x-z$ plane. The angular deviation of this vector from the $x-z$ plane, and the spacecraft's mean angular motion are used to compute a correction to the time estimate. The corrected time is used to re-propagate the orbital elements and re-position the spacecraft. This iteration continues until the time correction becomes less than the time between successive scans. The iteration converges at the solution time $t$, when $\mathbf{L}(t)$ is in the $x-z$ plane. At this time the scan line containing the appropriate FOV can be determined from $t$ and the time of first scan of the data. The correct sample number is calculated from the scan angle, defined as the angle between $\mathrm{L}(t)$ and the $y-z$ plane.

Usually the algorithm requires 4 or 5 iterations to reach convergence. We have found that it is generally sufficient to determine the scan line, sample number for every eighth pixel on every eighth row of the output image. The FOVs of the internal pixels can then be calculated by interpolation. The navigation package includes the interpolation procedure as an option, as it can result in a mis-registration of about 1 FOV. The error is apparent only for features containing very fine spatial structure. The interpolation option speeds up the registration significantly and is useful for qualitative "first looks" at the data.

\section{APPLICATIONS}

As stated in the introduction, the previously discussed algorithms have been implemented in a navigation package used to produce images of AVHRR data. This software requires three types of input: an ephemeris data set giving the most current orbital elements, the raw or calibrated AVHRR data, and a geographic data base used to create overlays of geographic features such as coastlines and rivers. The AVHRR data can be either HRPT (High Resolution Picture Tranmission), LAC (Local Area Coverage) or GAC (Global Area Coverage) data. The geographic data base currently used is the CIA World Data Base II or World Data Base I (Anderson and others, 1973).

Accurate image registrations require corrections for the roll, pitch, and yaw. An attitude drift of $0.2^{\circ}$ from the nominal value can result in a registration error of 2-7 FOVs (Brunel and Marsouin, 1987). If these quantities are unknown, then they must be determined. A desirable feature of the navigation system is the ability to determine and provide corrections for these attitude parameters. The full description of this method can be found in Rosborough and others (in preparation).

Briefly, the determination of the corrections requires the calculation of offsets of recognizable features in the image from the corresponding features of the map overlay. The features, referred to above as GCPs, can be coastlines, rivers, lakes, etc. At least two GCP are necessary to determine roll, pitch and yaw. It should be obvious that this procedure cannot be applied to data which contain no land. An application of this procedure is discussed later.

The navigation package allows for the creation of images in either a satellite perspective (forward navigation) or a geo-registered perspective (indirect navigation). An image created in satellite perspective will have samples oriented horizontally and scan lines vertically along the ground track of the satellite. The image can be either full resolution or sub-sampled. A distorted geographic overlay is created by using the indirect navigation routine to map the geographic latitude and longitude points of the data base to the corresponding scan line and sample of the output image.

As stated before, an image in the geo-registered perspective is created by specifying an output image size, a center latitude and longitude, a geographic projection type and a latitude range. Each pixel in the output image is mapped to a latitude and longitude which are then input into the indirect navigation algorithm to calculate the corresponding scan line and sample in the satellite data.

An example of an image created in satellite perspective is shown in Figure 2a. This is an HRPT NOAA 11 channel $2(0.9 \mu \mathrm{m})$ image. The contrast of the 
image has been stretched to accentuate the land-water differences. The data are from an ascending pass taken on 13 January 1992 at 1447:49 GMT. The image is 512 by 512 pixels and is centered on $7^{\circ} \mathrm{N}, 1^{\circ} \mathrm{E}$. It depicts a region of the Ghana coast of Africa. The western boundary is sample number 567 and the eastern boundary is sample 1078. The southern and northern boundaries are scan line 290 and 801 respectively. The scan line numbers are relative to the time of first scan mentioned above. This region was chosen for the extensive coast line and also because the image contains the nadir and is thus the highest resolution possible. The
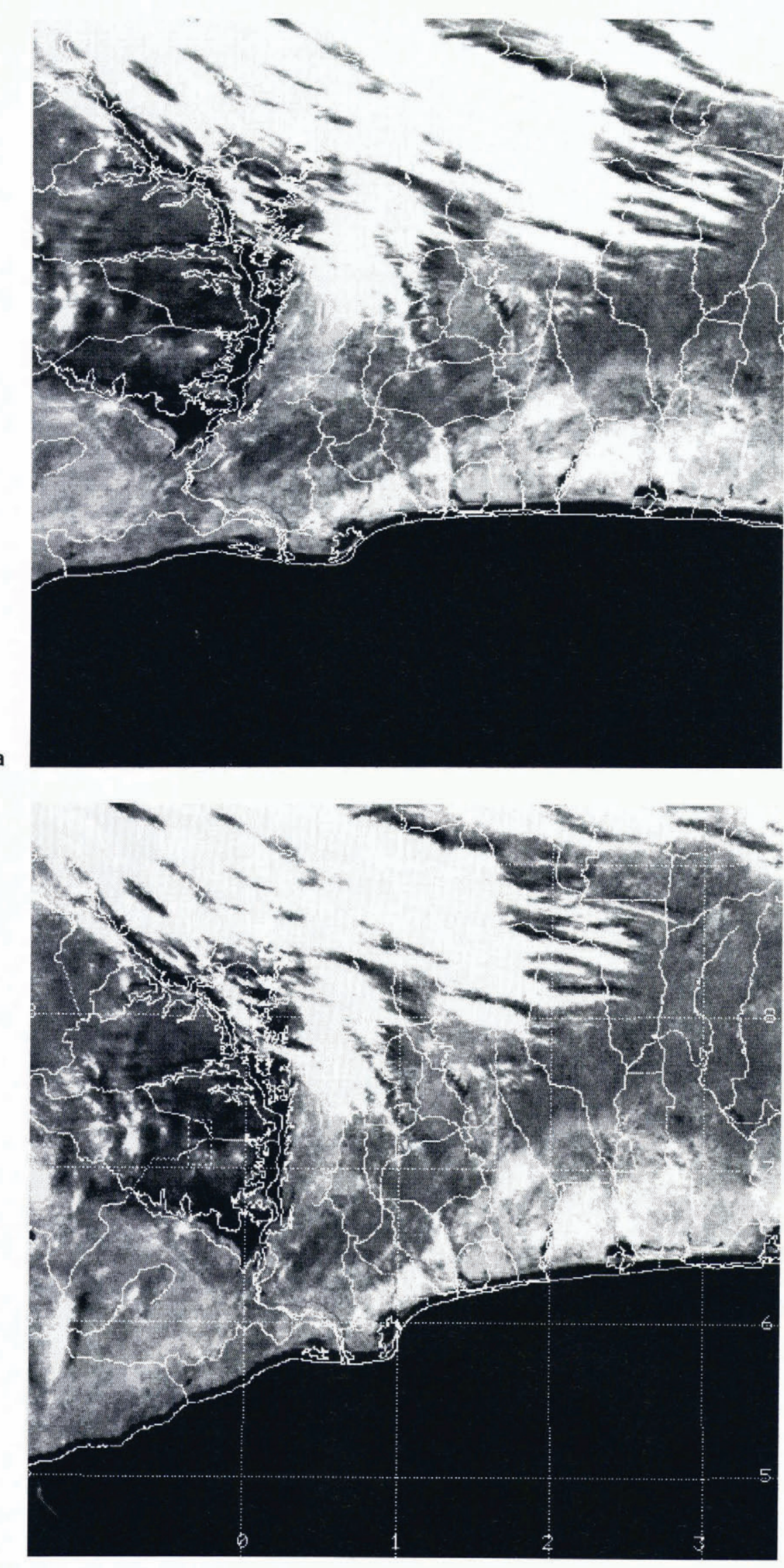


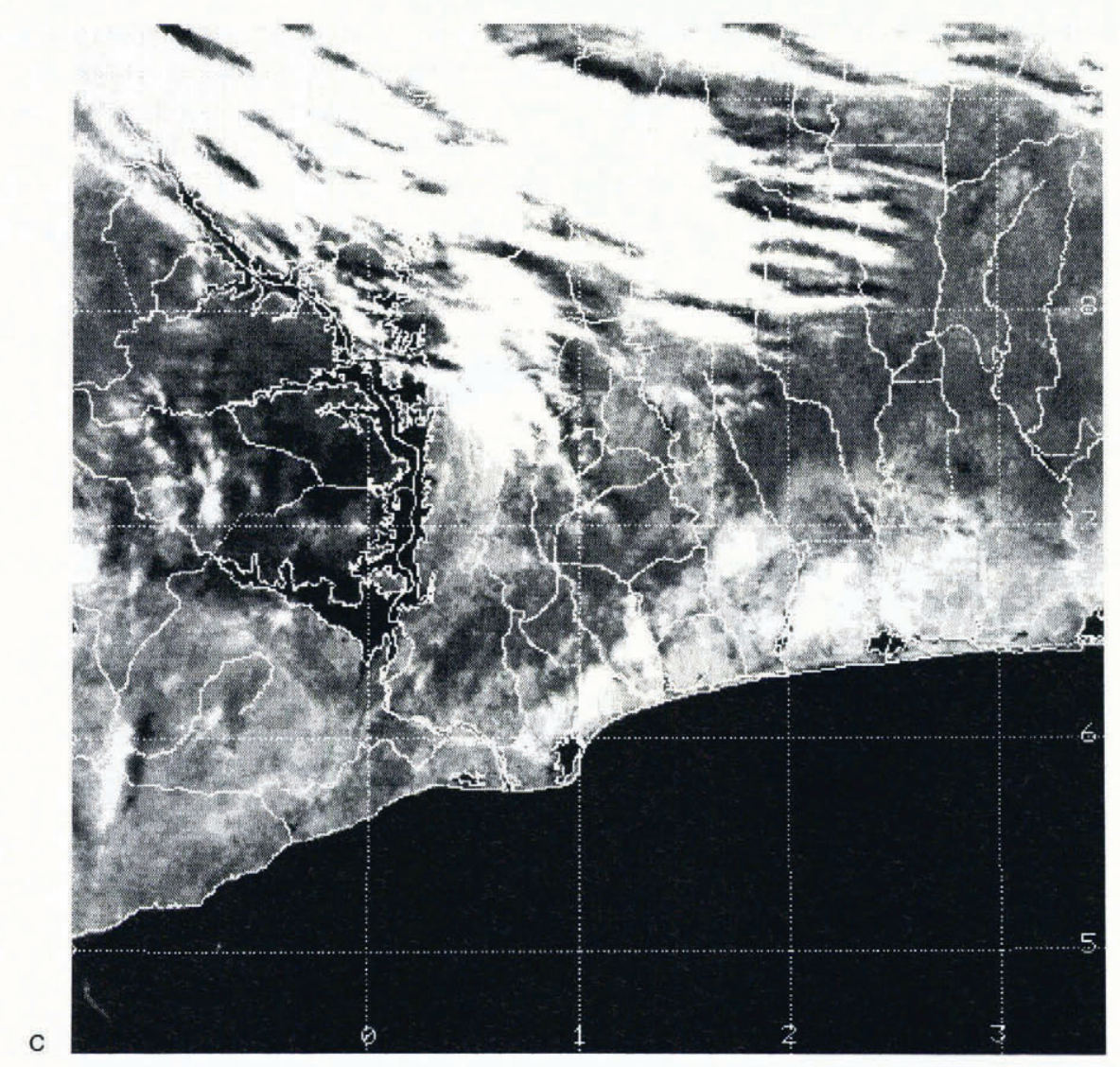

Fig. 2. (a) Satellite perspective image NOAA-11 near-IR channel showing registration errors. 13 January 1992, 1447:49UT. Centered on $7^{\circ} \mathcal{N}, 1^{\circ}$ E. (b) Geo-registered, NOAA-11 near IR channel image created by indirect navigation technique with no attitude corrections. Showing registration errors. 13 January 1992, 1447:49 UT. Centered on $7^{\circ} \mathcal{N}, 1^{\circ} \mathrm{E}$. (c) Geo-registered, NOAA-11 near-IR channel image createdby indirect navigation technique with attitude corrections. showing no registrat-ion errors. 13 January 1992, 1447:49UT. Centered on $7^{\circ} \mathcal{N}, 1^{\circ} E$.

image is made from the highest eight bits of the 10-bit raw data.

The offset of the image from the overlay is the result of a registration error caused by some combination of the following parameters: non-zero values for the spacecrafts roll, pitch and yaw, an error in the time of the first scan line of data, or errors resulting from inaccuracies in the positioning of the satellite. It is very difficult to identify the individual contributions the above sources make to the total error. For instance the registration error resulting from an offset in the time of first scan is very similar to the error caused by a non zero value for the pitch of the spacecraft. The present study has found that it is sufficient to compute an "effective" roll, pitch and yaw with the realization that these values correct errors from several unrelated sources. An example of an attitude corrected registration is presented later in the discussion. The typical registration error in Figure 2a is approximately $6 \mathrm{~km}$.

The corresponding image in the geo-registered perspective is presented in Figure 2b. The image was created using the same data as Figure $2 \mathrm{a}$ and is centered on the same latitude and longitude. It is $512 \times 512$ pixels in size and the geographic projection is conic with a latitude range of $5^{\circ}$. This combination results in an image which is as close to full resolution as is possible with georegistration. Note the different perspective and orientation of the geographical features as compared with Figure 2a.

As stated in the introduction, an advantage of the georegistered perspective is the co-location of pixels which allows for composites and comparisons of different orbits. This is not possible with a satellite perspective image. Note also the same registration errors that were present in Figure 2a.

Correction for the effective roll, pitch and yaw requires three steps. First, an image and overlay are created with no attitude corrections. The image can be in either of the two perspectives discussed above. Second, the offsets of ground control points in the image from the overlay must be determined and used to compute the attitude parameters. The geographical position of the GCPs should be determined with an accuracy of approximately $1 \mathrm{~km}$. As discussed above, at least two of these points are necessary. Third, the image is then renavigated using the correction.

The determination of the attitude parameters is more accurate if the ground control points are widely separated with respect to the line and sample numbers. We have found that accurate results are obtained when one GCP is 
at one "corner" of the dataset and the other close to the opposite corner. When the effective corrections are determined in this manner, they will accurately correct any images made from that data. In other words, a given AVHRR dataset consisting of 5400 scan lines (15 min of data) may contain several different regions of interest. Each region may require an image characterized by a particular size and projection. Once the effective correction is determined for the dataset, the individual images can be accurately registered without the need for further GCP analysis.

Figure 2c shows the corresponding geo-registered image with corrections for the effective attitude parameters. Two GCPs were used to obtain the attitude correction. Both of the GCPs are from regions outside of the image. One GCP is the coastline of one of the Canary Islands located at $28.125^{\circ} \mathrm{N}, 15.678^{\circ} \mathrm{W}$. This point is located in the northwest corner of the dataset. The second GCP, located in the southeast corner of the dataset, is the coastline of Malabo Island at $3.75^{\circ} \mathrm{N}, 8.734^{\circ} \mathrm{E}$.

In Figure 2c, the correspondence between the overlay and the image shows that the registration is accurate to within a pixel; note that this accuracy holds for any portion of the dataset. For these data, the values for the effective roll, pitch and yaw were $-0.10^{\circ}, 0.51^{\circ}$, and $0.05^{\circ}$ respectively. Again, these are not the true values of the roll, pitch and yaw, but instead are corrections which contain the true values. The clock on board the TIROS spacecraft has a systematic drift which can cause an error in the timing of the data. The fact that the pitch is the largest of the correction parameters indicates that timing is probably one of the largest sources of registration error.

\section{MODEL COMPARISON}

As stated earlier, the orbital model used assumes that the

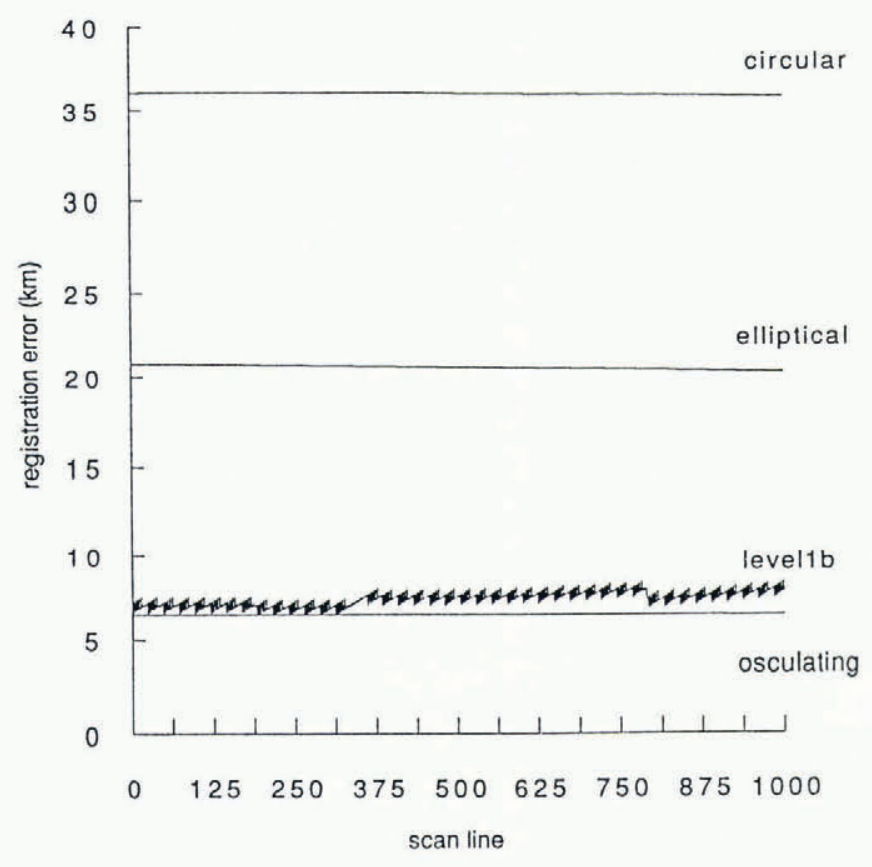

Fig. 3. Nadir registration error along track for different orbital models. orbit is a secularly precessing decaying ellipse with corrections for both the short period osculating perturbations and attitude parameters. This model represents a progression in complexity which begins with a simple circular model and evolves to the model described above. The progression can be characterized as four separate models: circular, elliptical, elliptical with osculating corrections, and finally elliptical with both osculating and attitude corrections.

We felt it worthwhile to compare the accuracies of the above components with respect to image navigation. Figure 3 depicts the results of the comparison which was constructed using the same AVHRR data discussed earlier. The latitude and longitude of the nadir point was determined using the elliptical, osculating model with attitude corrections. These latitude and longitudes were taken to be "ground truth" since a geo-registered image created by this model has a very close correspondence with the map overlay. The above components were added cumulatively to the circular model and the difference in $\mathrm{km}$ between the predicted nadir point and the groundtruth nadir point was computed and plotted for every scanline $(0.167 \mathrm{~s})$ for 1000 scanlines. The ephemeris epoch for this day was at $0000 \mathrm{UT}$, so the orbital predictions were made approximately $14 \mathrm{~h}$ from epoch.

The commonly used NOAA Level lb format (NOAA, 1991) for AVHRR data supplies a latitude and longitude for every 40 samples on each scan line. The nadir point latitude and longitude were extracted from the Level $1 \mathrm{~b}$ data and also used in the comparison. As expected, the circular model resulted in the highest error, this being above $35 \mathrm{~km}$. The circular model was created by initializing and keeping the orbital eccentricity at 0 . Adding the correct eccentricity, but keeping it constant throughout the $167 \mathrm{~s}$ reduced the error to $20 \mathrm{~km}$. Adding the short period osculating corrections resulted in an error of about $6 \mathrm{~km}$. This error is very close to the error obtained using the Level $\mathrm{lb}$ navigation. It must be noted that the $6 \mathrm{~km}$ error associated with the osculating model contains the errors from all sources, including time and attitude errors. As stated earlier, timing errors due to clock drift are a significant component of the total error.

\section{CONCLUSIONS}

The problem of precise AVHRR image navigation is very tractable in theory, but has proven to be somewhat difficult in practice. The difficulties have arisen due to several of the areas discussed in this presentation; inaccuracies in orbit modeling, error in timing, unknown attitude parameters, etc. By systemizing the procedures involved in image navigation we have gained a better understanding of how to correct for the cumulative effect of the various error components. This correction has resulted in a navigation system capable of geo-registering a satellite image to within $1 \mathrm{~km}$.

The favorable results obtained from this study have spawned a follow-up project (in progress) which is investigating the possibility of modelling the correction parameters. Satellite clock corrections (supplied by NOAA) will be appropriately applied to remove timing from the sources of registration error. The resulting net 
error is expected to exhibit systematic variations which can be mathematically described over some period of time. Success in this endeavor will enable a more automatic approach to image navigation and will also benefit researchers whose data are lacking in identifiable GCPs. Also, the resulting error will be compared with the roll, pitch and yaw values which are transmitted as part of the HRPT data stream.

\section{REFERENCES}

Anderson, D. E., J. L. Angel and A. J. Gorny. 1973. World Data Bank II: content, structure and application. Washington, DC, Central Intelligence Agency. Office of Geographic and Cartographic Research.

Brouwer, D. 1959. Solution of the problem of artificial satellite theory without drag. Astron. F., 64, 378-397.

Brunel, P. and A. Marsouin. 1987. An operational method using Argos orbital elements for navigation of AVHRR imagery. Int. F. Remote Sensing, 8, 569-578.
Brush, R.J.H. 1982. A real time data retrieval system for images from polar orbiting satellites. (Ph.D. thesis, University of Dundee.)

Brush, R.J.H. 1987. The navigation of AVHRR imagery. Int. F. Remote Sensing, 9, 1491-1502.

Emery, W.J. and M. Ikeda. 1984. A comparison of geometric correction methods of AVHRR imagery. Can. J. Remote Sensing, 10, 46-56.

Emery, W. J., J. Brown and Z. P. Nowak. 1989. AVHRR image navigation: summary and review. Photogramm. Eng. Remote Sensing, 55, 1175-1183.

Ho, D. and A. Asem. 1986. NOAA AVHRR image referencing. Int. F. Remote Sensing, 7, 895-904.

Kidwell, K.B., ed. 1991. NOAA polar orbiter data; users' guide. Washington, DC, National Oceanic and Atmospheric Administration.

The accuracy of references in the text and in this list is the responsibility of the authors, to whom queries should be addressed. 